Received: 2018.03.19

Accepted: 2018.05.21

Published: 2018.09 .19

\title{
Phenotype of Vascular Smooth Muscle Cells (VSMCs) Is Regulated by miR-29b by Targeting Sirtuin 1
}

Authors' Contribution:

Study Design A

Data Collection B

Statistical Analysis C

Data Interpretation D

Manuscript Preparation E

Literature Search F

Funds Collection G

Corresponding Author:

Source of support:

Background:

Material/Methods:

Results:

Conclusions:

MeSH Keywords:

Full-text PDF:
Kun Fang, e-mail: fangkun1010@163.com

Departmental sources
1 Center for Medical Genetics, School of Life Sciences, Central South University, Changsha, Hunan, P.R. China

2 Department of Vascular Surgery, Fuwai Hospital, Chinese Academy of Medical Science and Peking Union Medical College, National Center for Cardiovascular Diseases, Beijing, P.R. China
CDF 2 Xiong Zhang

CDE 2 Kun Fang

Phenotypic switch of vascular smooth muscle cells (VSMCs) participates in the etiology of various vascular diseases. It has been proved that microRNAs (miRNAs) serve as crucial regulators of functions of VSMCs. This study aimed to discover how miR-29b regulates the transformation of VSMCs phenotypes in mice.

Primary VSMCs of aorta in mice were cultured in DMEM medium. A series of experiments involving transfection of oligonucleotides in cultured VSMCs, quantitative reverse transcription PCR (qRT-PCR), luciferase reporter assay, and Western blotting analysis were performed in this study.

We found that in VSMCs cultured in presence of stimulator, platelet-derived growth factor-BB (PDGF-BB), miR-29b was upregulated significantly and expressions of VSMC-phenotype-related genes ( $\alpha$-SMA, calponin, and SM-MHC) were regulated by miR-29b. Moreover, through downregulation of sirtuin 1 (SIRT1), miR-29b affects phenotypic transformation of VSMCs. Luciferase report assay identified a significant increase of SIRT1 3'-UTR activity in treatment with miR-29b inhibitor, which, however, was reversed in the presence of miR-29b mimic. Suppression of miR-29b reversed the activation of NF-KB induced by PDGF-BB in VSMCs.

We concluded that miR-29b is an important regulator in the PDGF-BB-mediated VSMC phenotypic transition by targeting SIRT1. Interventions aimed at miR-29b may be promising in treating numerous proliferative vascular disorders.

Dementia, Vascular • Sex • Sirtuin 1

https://www.medscimonit.com/abstract/index/idArt/910068

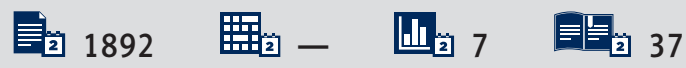




\section{Background}

Vascular smooth muscle cells (VSMCs) are a group of specialized cells characterized by remarkable plasticity in response to various environmental cues in mature cells [1,2]. Vascular damage usually comes with alterations in phenotype, ranging from a contractile differentiated state to the dedifferentiated state characterized by proliferation and migration $[3,4]$. This response participates in the development of vessel disorder as well as remodeling, which includes aggregation of dedifferentiated arterial VSMCs, and participates in the etiology of various vessel disorders, including arteriosclerosis [5,6], which prompted us to further investigate the etiology of the VSMC phenotype.

In response to vascular damages, release of a variety of factors is induced to activate VSMCs proliferation. For instance, upregulation of PDGF-BB can initiate the associated signal pathway to activate the proliferation of VSMCs, so as to complete the phenotypic transition from differentiated status to dedifferentiated status. Through binding to its receptor (PDGFR- $\beta$ ), PDGF-BB can further trigger a variety of signal cascades, including silent information regulator transcript-1 (SIRT1), PI3K/AKT, ERK, and MAPK pathways in vascular injury. SIRT1 is a nicotinamide adenine dinucleotide (NAD)-dependent nuclear histone deacetylase that mainly plays an anti-inflammatory role in the vasculature by regulating proliferation and the cell cycle. One of its major deacetylation targets is nuclear factor- $\mathrm{KB}$ (NF-KB). The acetylation of NF-KB and its proinflammatory program can lead to the induction of CyclinD1 and MMP-9 in the vasculature, which is associated with VSMC proliferation and migration. Recent studies show that PDGFR- $\beta /$ SIRT-1/NF-KB signaling plays a role in the VSMC phenotypic transformation after subarachnoid hemorrhage [6-8].

Emerging evidence indicates that a number of new classes of non-coding RNAs have been discovered in regulating tissue homeostasis under different conditions $[9,10]$. The miRNAs are non-coding RNAs that regulate the expression level after transcription via binding to target mRNAs, thus suppressing translation or stimulating degradation of mRNAs [11]. It has also been revealed that miRNAs are associated with SMC plasticity [12-14]. A number of miRNAs, such as miR-21, miR-206, miR-124, miR-199, and miR-182, have been recognized as regulators of the VSMC phenotype, not only in vivo, but also in vitro [15-19]. Changes in expression of miRNAs are show promise in providing novel treatment strategies.

As a tumor suppressor, miR-29b represses migration and invasion of malignant cells by directly targeting lysyl oxidaselike 2 genes [20]. It is reported that miR-29b plays a role in accelerating the apoptotic events in smooth muscle cells, which is mediated byMMP-2. Moreover, in presence of miR-29b, migration and proliferation of VSMCs are also suppressed during neointimal generation. Nevertheless, few studies have elucidated the significance of miR-29b in phenotypic switch of VSMCs. Thus, we aimed to discover how miR-29b works in VSMCs phenotypic switch.

\section{Material and Methods}

\section{Cell culture and treatment}

Primary VSMCs of the aorta in mice (CHI Scientific, Jiangyin, China) were cultured in DMEM supplemented with fetal bovine serum (10\%; Invitrogen, Carlsbad, CA, USA) in $5 \%$ CO2 and at $37^{\circ} \mathrm{C}$, and, after not more than 8 passages, were treated by PDGF-BB (Rocky Hill, USA) for $24 \mathrm{~h}$.

\section{Transfection of oligonucleotides in cultured VSMCs}

For miR-29b knockdown, SMCs at $2 \times 10^{5} /$ well were cultured in 6-well plates for $24 \mathrm{~h}$, followed by transfection of miR-29b inhibitor (GenePharma, $80 \mathrm{nM}$ ) or negative control ( $80 \mathrm{nM}$ ) using Lipofectamine 2000 (Invitrogen, Carlsbad, USA). Transfected cells were further cultured in antibiotics-free medium for $24 \mathrm{~h}$ for subsequent experiments. For miR-29b overexpression, procedures were the same as the previous protocol, but the transfection was carried out with miR-29b mimic (GenePharma, $50 \mathrm{nM}$ ) or negative control (50 nM). Sirt1 knockdown was performed using siRNA (GenePharma) or the control ( $40 \mathrm{nmol} / \mathrm{L}$ ), followed by transfection using Lipofectamine ${ }^{T M}$ RNAiMAX.

\section{Quantitative reverse transcription PCR (qRT-PCR)}

Isolation of total RNA from murine aortic VSMCs was performed with TRIzol$^{\oplus}$. The qRT-PCR of U6 and miR-29b was carried out by using the TaqMan ${ }^{\circledast}$ microRNA assay kit. Primers that were used to measure the levels of calponin, SM-MHC, and $\alpha$-SMA are listed as follows:

$\alpha$-SMA: forward primer: 5'-GTCCCAGACATCAGGGAGTAA-3', reverse primer: 5'-TCGGATACTTCAGCGTCAGGA3';

Calponin: forward primer: 5'-TCTGCACATTTTAACCGAGGTC-3', reverse primer: 5'-GCCAGCTTGTTCTTTACTTCAGC-3';

SM-MHC: forward primer: 5'-AAGCTGCGGCTAGAGGTCA-3', reverse primer: 5'-CCCTCCCTTTGATGGCTGAG-3'; GAPDH: forward primer: 5'-GCAAGTTCAACGGCACAG-3', reverse primer: 5'-GCCAGTAGACTCCACGACATA-3.

The relative expression miR-29b to U6, calponin, SM-MHC, $\alpha-S M A$, and GAPDH was identified using the $2^{-\Delta \Delta} \mathrm{Ct}$ method.

\section{Luciferase reporter assay}

After co-transfection of miR-29b or its inhibitor in the presence of lentiviral $\mathrm{PGL} 3$ vector containing clones of relevant 


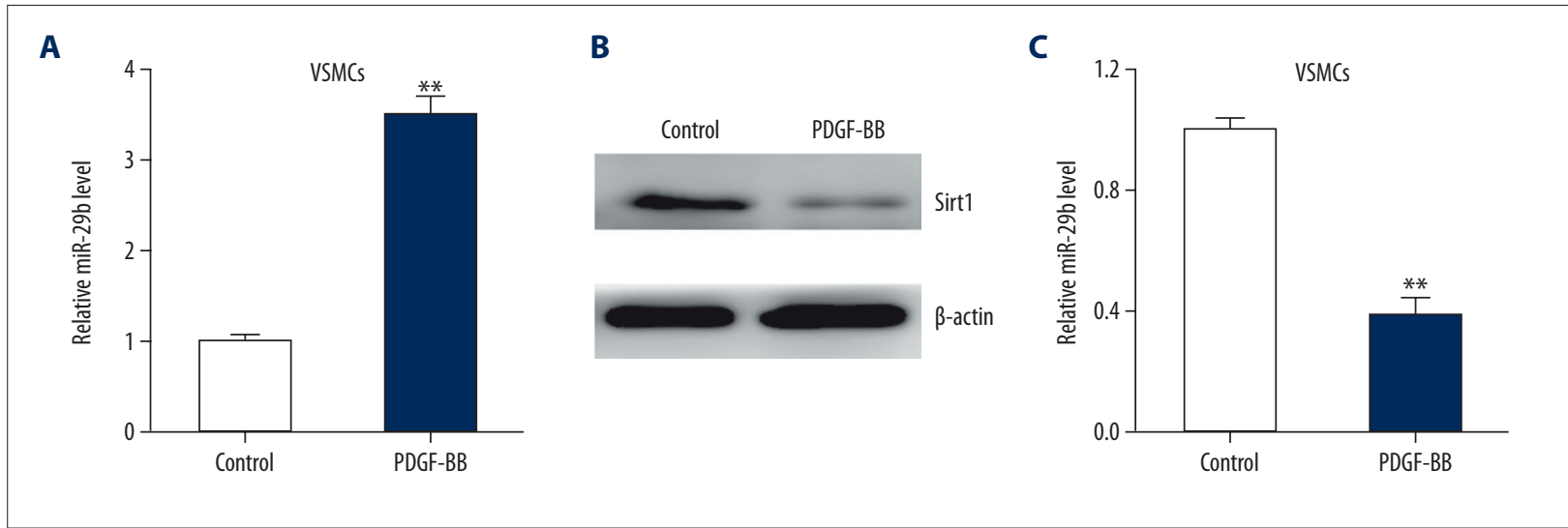

Figure 1. miR-29b is upregulated in PDGF-BB-treated VSMCs. (A) mRNA expression of miR-29b in PDGF-BB-treated (20 ng/ml) VSMCs for 24 h. (B-C) Representative immunoblots (B) and quantitative analysis of Sirt1 (C) in PDGF-BB-treated VSMCs. This procedure was performed in triplicate with data expressed as means \pm S.E.M. ${ }^{* *} p<0.01$ vs. control group.

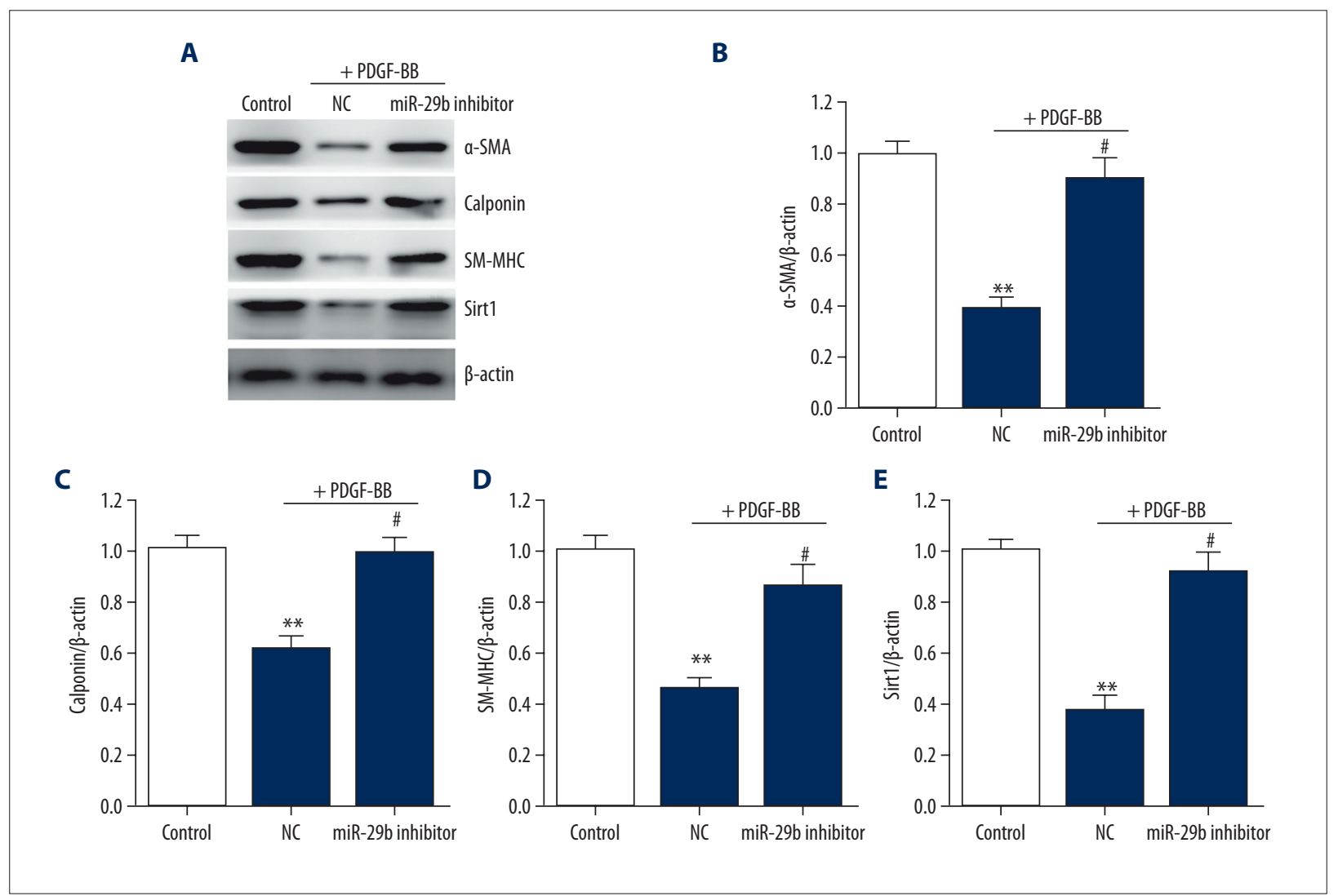

Figure 2. miR-29 knockdown increases the differentiation marker genes expression in VSMCs. (A-E) Representative immunoblots (A) and quantitative analysis of $\alpha$-SMA (B), calponin (C), SM-MHC (D), and Sirt1 (E) in VSMCs. This procedure was performed in triplicate with data expressed as means \pm S.E.M. ${ }^{* *} p<0.01$ vs. control group, ${ }^{*} p<0.05$ vs. negative control group.

sequences of SIRT1, we detected the activities of relevant luciferases in the luciferase reporter assay system.

\section{Western blotting (WB) analysis}

Homogenization was carried out using lysis buffer (Beyotime, China). Proteins were then loaded for electrophoresis, and those proteins on gel that were transferred on the PVDF membrane (Millipore, MA, USA) were blocked by $5 \%$ skimmed-milk for $1 \mathrm{~h}$. 
A

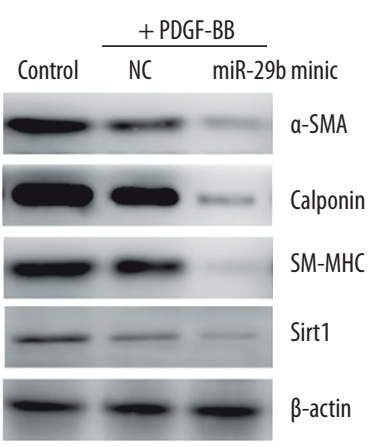

B

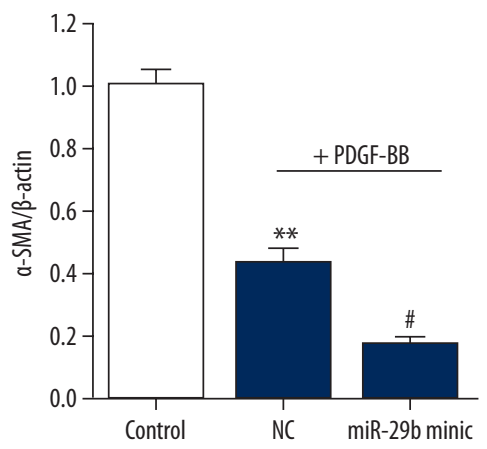

C

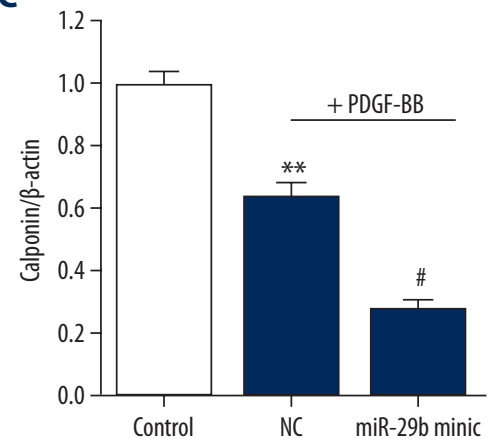

D

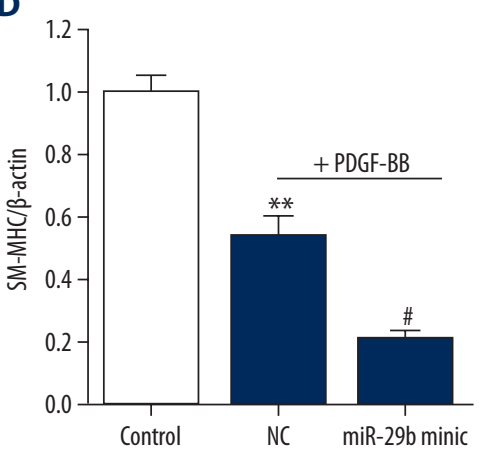

E

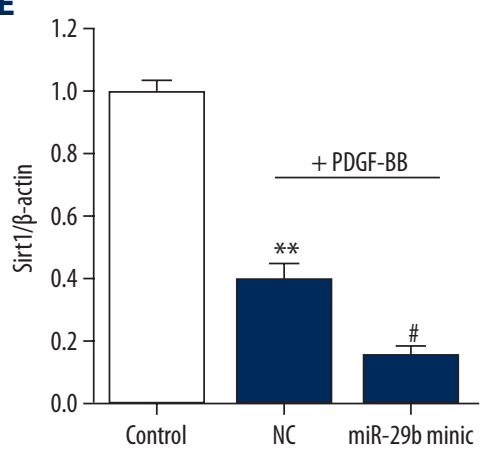

Figure 3. Overexpression of miR-29 downregulates differentiation marker in VSMCs. (A-D) Representative immunoblots (A) and quantitative analysis of $\alpha$-SMA (B), calponin (C), SM-MHC (D), and Sirt1 (E) in VSMCs. This procedure was performed in triplicate with data expressed as means \pm S.E.M. ${ }^{* *} p<0.01$ vs. control group, ${ }^{*} p<0.05$ vs. negative control group.

Proteins on the membrane were incubated with anti-SM-MHC (1: 2000), anti-Sirt1 (1: 500), anti- $\alpha$-SMA (1: 1000), anti-calponin (1: 1000), and anti- $\beta$-actin (1: 5000) antibodies (CST, MA, USA) overnight at $4^{\circ} \mathrm{C}$, and then incubated with HRP-conjugated secondary antibodies. Analysis of protein expression was carried out with bands on membranes using ECL reagent (Pierce, IL, USA). The data are from 3 independent experiments.

\section{Statistical analysis}

The data are listed in the form of mean \pm S.E.M. ANOVA and Tukey's post hoc test were applied for evaluation of differences. For the evaluation, $\mathrm{P}$ values $<0.05$ were considered as significant.

\section{Results}

\section{miR-29b is upregulated in the PDGF-BB-treated VSMCs}

To investigate how miR-29b modulates the phenotype of VSMCs, we treated the VSMCs with PDGF-BB. qRT-PCR results of miR-29b showed that PDGF-BB treatment upregulated miR-29b in comparison with control cells (Figure 1A) and SIRT1 level was decreased when compared with cells in the control group (Figure 1B, 1C), suggesting a correlation between upregulation of miR-29b and VSMCs phenotypic modulation.

\section{Knockdown of miR-29 suppresses the transformation of VSMC phenotypes}

To elucidate how miR-29b affects the transformation of VSMC phenotypes, we transfected VSMCs with miR-21b inhibitor or negative control to simulate functional loss for $24 \mathrm{~h}$, followed by stimulation using PDGF-BB for $24 \mathrm{~h}$. Differentiation-related genes, includinga-SMA, SM-MHC and calponin, and Sirt1, in VSMCs were downregulated, indicating that transfection of miR-29b inhibitor abolished changes in these genes and in Sirt1 (Figure 2).

\section{miR-29 promotes the transformation of VSMC phenotypes}

To further assess how miR-29b works in transformation of VSMC phenotypes, we then transfected miR-29bmimic or negative control into VSMCs to regain the functions; $24 \mathrm{~h}$ later, cells were incubated with PDGF-BB for another $24 \mathrm{~h}$. Treatment 


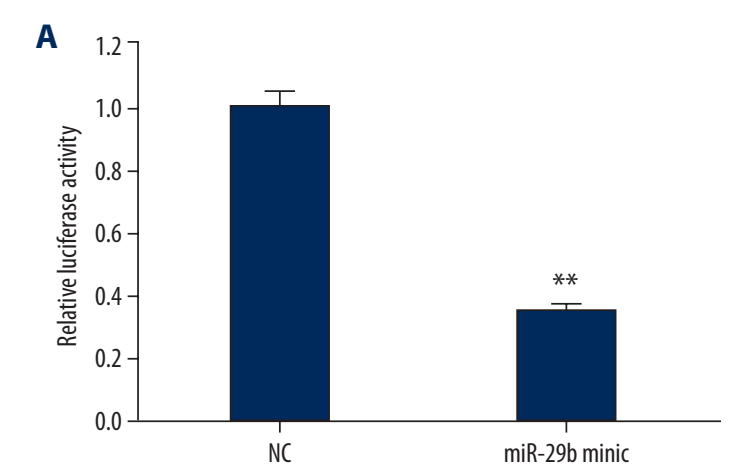

B
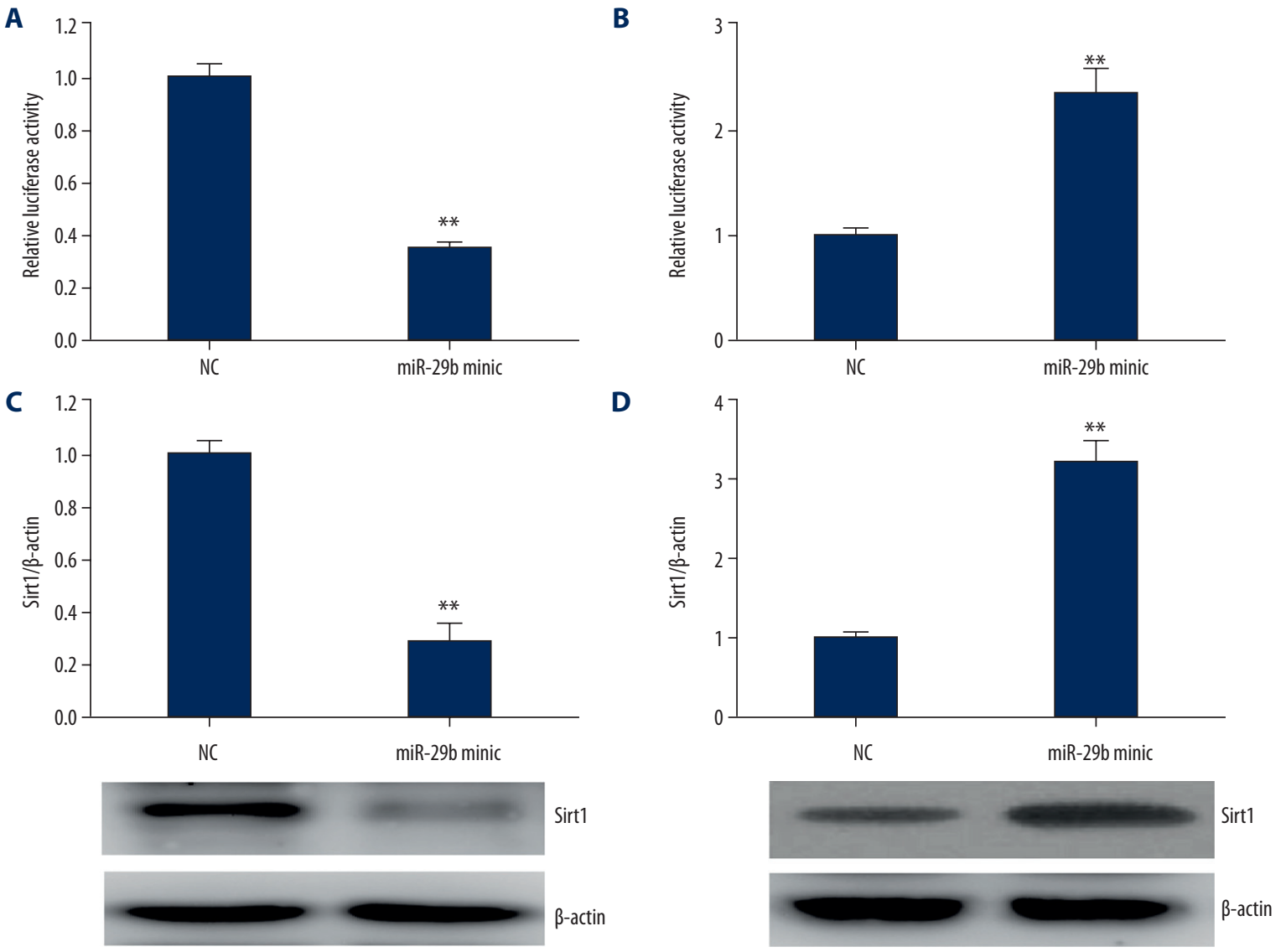

Figure 4. Sirt1is a potential target gene of miR-29b. (A, B) mRNA expressions of miR-29b andSirt1 determined by luciferase activity assay. (C, D) Representative immunoblots and quantitative analysis of Sirt1in miR-29b mimic- (C) or inhibitor (D)-transfected VSMCs for $24 \mathrm{~h}$. This procedure was performed in triplicate with data expressed as means \pm S.E.M. ${ }^{* *}$ p $<0.01$ vs. NC group.

of PDGF-BB notably reduced the levels of Sirt1 and VSMCs differentiation marker genes (Figure 3). Transfection of miR-29b mimic enhanced the effect PDGF-BB on reducing the expression of these genes and Sirt1. Taken together, our results show that miR-29b is involved in the transformation of VSMC phenotypes as a new intermediate.

\section{Sirt1is a target of miR-29b}

The target genes of miR-29b were analyzed using Target Scan. Sirt1 was selected because it participated in the modulation of alteration in phenotype of VSMCs [21]. The results of luciferase reporter assay showed that the luciferase activity was remarkably reduced when cells were transfected with Sirt1-3' UTR and miR-29b, while it was noticeably increased when cells were transfected with Sirt1-3' UTR and miR-29b suppressor (Figure 4A, 4B). The above findings show that Sirt1 mRNA is directly targeted by miR-29b. Furthermore, the findings revealed that miR-29b can reversibly modulate levels of Sirt1 proteins in cultured VSMCs (Figure 4C, 4D).

\section{Knockdown of Sirt1 reverses the effect of miR-29b inhibition on VSMC phenotypic switch.}

To verify the role of SIRT1 in miR-29b-regulated VSMCs phenotypic modulation, we transfected si-Sirt1 and miR-29b inhibitor into VSMCs. As shown in Figure 5, miR-29b inhibitor increased the expression of VSMC differentiation marker genes, which were partially reversed by Sirt1 knockdown, suggesting that the regulatory role of miR-29b in transition of VSMCs phenotype depends on inhibition of Sirt1.

\section{Knockdown of miR-29b suppresses activation of NF- $\kappa B$}

NF-KB signaling pathway plays an important role in VSMCs phenotypic modulation [22]. We found that PDGF-BB significantly enhanced the activation of NF- $\mathrm{KB}$ and reduced the Sirt1 expression. miR-29 knockdown abrogated the enhancement of PDGF-BB on the activation of NF- $\mathrm{KB}$ and restored the Sirt1 level (Figure 6). Together, these results suggest that miR-29b 


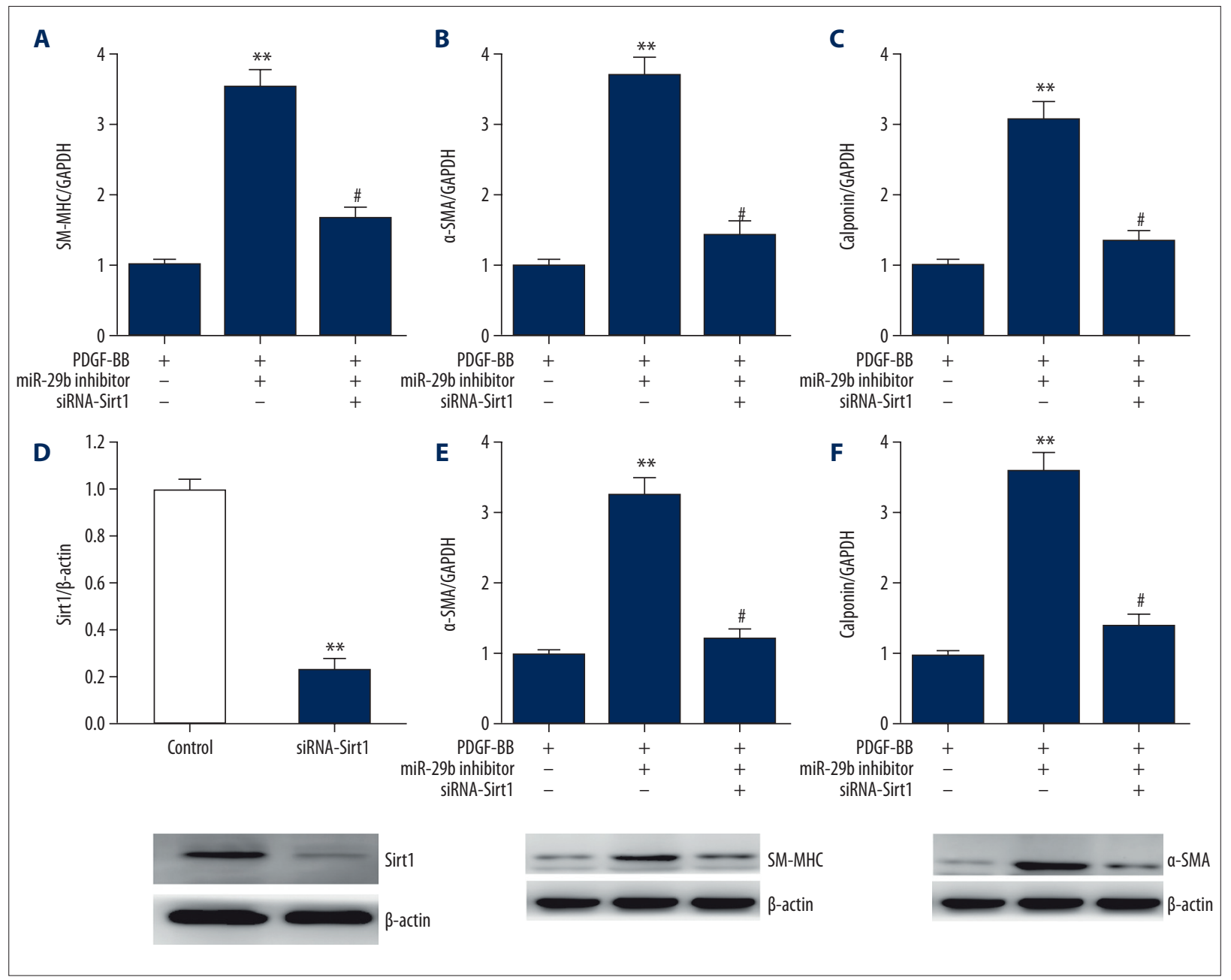

Figure 5. Knockdown of Sirt1 reverses VSMC phenotypic modulation caused by miR-29b inhibitor. A-C, mRNA expressions of SM-MHC (A), $\alpha$-SMA (B), and calponin (C) in VSMCs as determined using qRT-PCR. This procedure was performed in triplicate with data expressed as means \pm S.E.M., ${ }^{* *} p<0.01$ vs. PDGF-BB group, ${ }^{*} p<0.05$ vs. miR-29b inhibitor group. (D) Representative immunoblots and quantitative analysis of Sirt1 in VSMCs transfected with Sirt1 siRNA. This procedure was performed in triplicate with data expressed as means \pm S.E.M. ${ }^{* *} p<0.01$ vs. control group. (E, F) Representative immunoblots and quantitative analysis of SM-MHC (E) and $\alpha$-SMA $(F)$ in VSMCs. This procedure was performed in triplicate with data expressed as means \pm S.E.M., ${ }^{* *} p<0.01$ vs. PDGF-BB group, ${ }^{*} p<0.05$ vs. miR-29b inhibitor group.

regulates VSMCs phenotypic modulation by suppressing the Sirt1/NF-kB signaling pathway.

\section{Discussion}

In the present study, miR-29b is involved in regulating the transformation of VSMCs transformation through the Sirt1NF- $\mathrm{KB}$ pathway. Results showed that miR-29b was upregulated in VSMCs treated by PDGF-BB, which further downregulated SIRT1. In the absence of miR-29b, VSMCs differentiation was enhanced, which was reversed by its overexpression. Sirt1 knockdown reversed the miR29b inhibitor, modulated VSMC differentiation marker genes expression, and promoted transformation of VSMC phenotype from a contractile to synthetic phenotype (Figure 7). Therefore, the miR-29b-Sirt1 pathway may be involved in the transformation of phenotypes in VSMCs as a possible target in treatment of vascular diseases.

Since the microenvironment of vessel walls varies over time, VSMCs display remarkable versatility. Under regular quiescent circumstances, primary activities of VSMCs include contraction to maintain blood flow. In altered microenvironments, various changes are stimulated in VSMCs to achieve the conditions of proliferation and synthesis. Plasticity of phenotype is crucial for the development of vessels. However, these abnormal alterations in the phenotype lead to generation and progression of different proliferative diseases of vessels, 


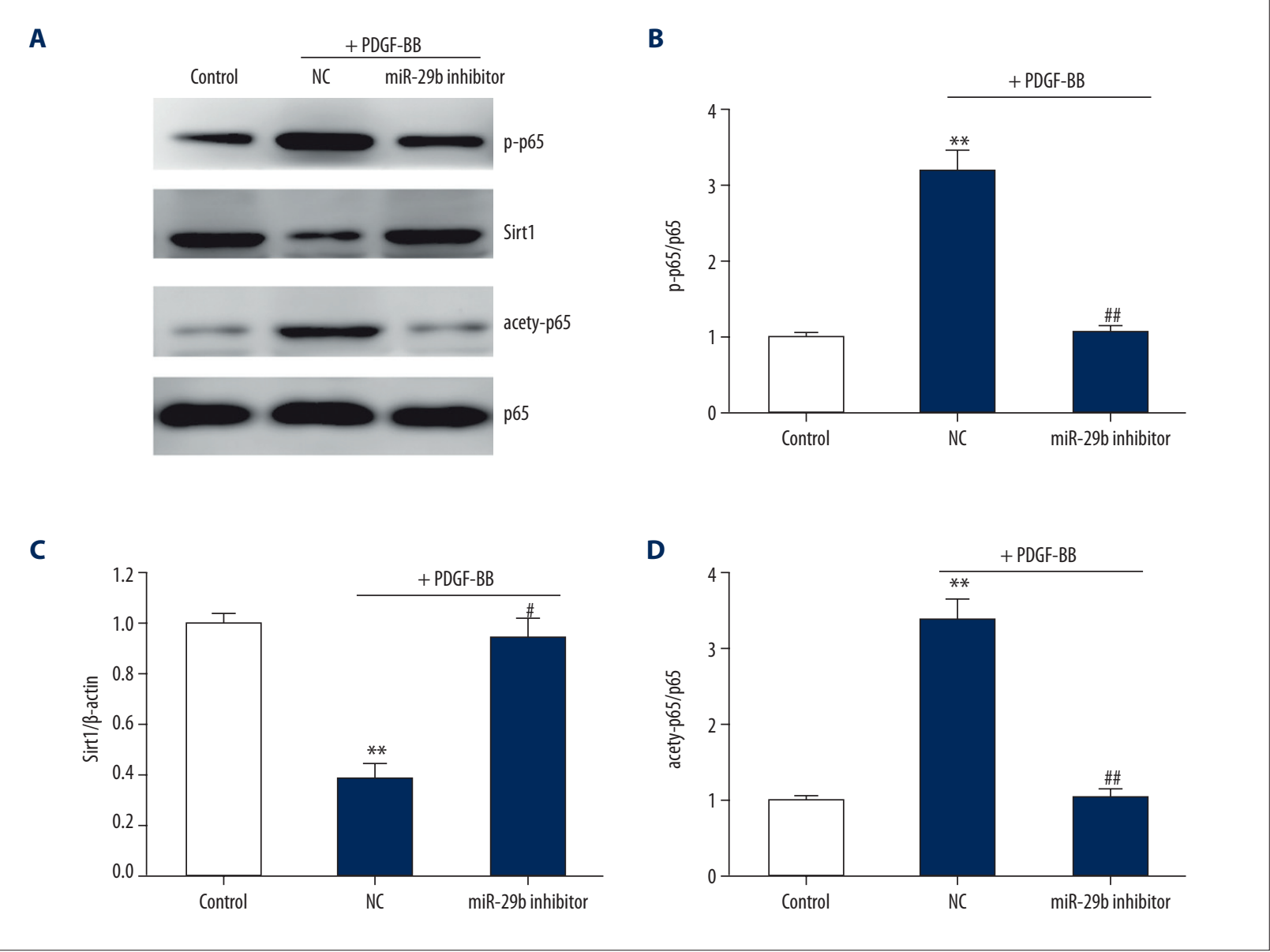

Figure 6. Knockdown of miR-29 suppresses NF-KB activation in VSMCs. (A-D) Representative immunoblots (A) and quantitative analysis of p-p65 (B), Sirt1 (C), and acetylation of p65 (D) in VSMCs. This procedure was performed in triplicate with data expressed as means \pm S.E.M., ${ }^{* *} p<0.01$ vs. control group, ${ }^{\#} p<0.05$ vs. negative control group.

including atherosclerosis, hypertension, and restenosis after angioplasty [23-26]. The etiology of these alterations in VSMC phenotype is still unclear. Recent reports have demonstrated that miRNAs participate in the regulation of the phenotype of VSMCs [27-29]. Selective expression of miR-145 occurs in murine VSMCs, which is remarkably inhibited during the alteration in phenotype triggered by PDGF-BB in vitro as well as in balloon-damaged murine arteria carotis. The miR-145 elimination noticeably attenuated the contractile state. Moreover, recovery of miR-145 concentration inhibits the dedifferentiation of VSMCs, as well as the generation of neointimal lesions that occur subsequent to vessel damage through suppressing KLF5 $[30,31]$. In the present study, miR-29b was significantly upregulated in PDGF-BB-treated VSMCs. Emerging evidence shows the key role of miR-29b in smooth muscle cell homeostasis. It is reported that miR-29b promotes apoptotic activity of smooth muscle cells through MMP-2. In addition, miR-29b inhibits the activities of VSMCs during neointimal generation, including cell migration and proliferation, but the significance of miR-29b in transformation of phenotype in VSMCS remains unknown. To clarify how miR-29b works in phenotypic switch of VSMCs induced by PDGF-BB, we knocked down miR-29b and found that genes relating to differentiation were upregulated to suppress PDGF-BB. In case of overexpression, miR-29b blocked the transformation of phenotype in VSMCs, suggesting the modulating effect of miR-29b on phenotypic transformation in VSMCs. However, the relevant mechanism remains obscure.

Some pathways regulate the alteration of VSMC phenotype [32-34]. Multiple studies have proved that Sirt1/NF-KB axis participates in the alteration of phenotype of VSMCs [35-37]. We found that miR-29b bound to the MRNA of SIRT1 in luciferase reporter system, suggesting that SIRT1 is the target of miR-29b, consistent with the results of previous bioinformatics analysis. miR-29b exerts a negative effect in regulating the level of SIRT in VSMCs. When miR-29b was knocked down, differentiation marker genes were upregulated, with an inhibitory effect on NF-KB. More importantly, in the absence of Sirt1, transformation of phenotype in VSMCs was reversed in those 


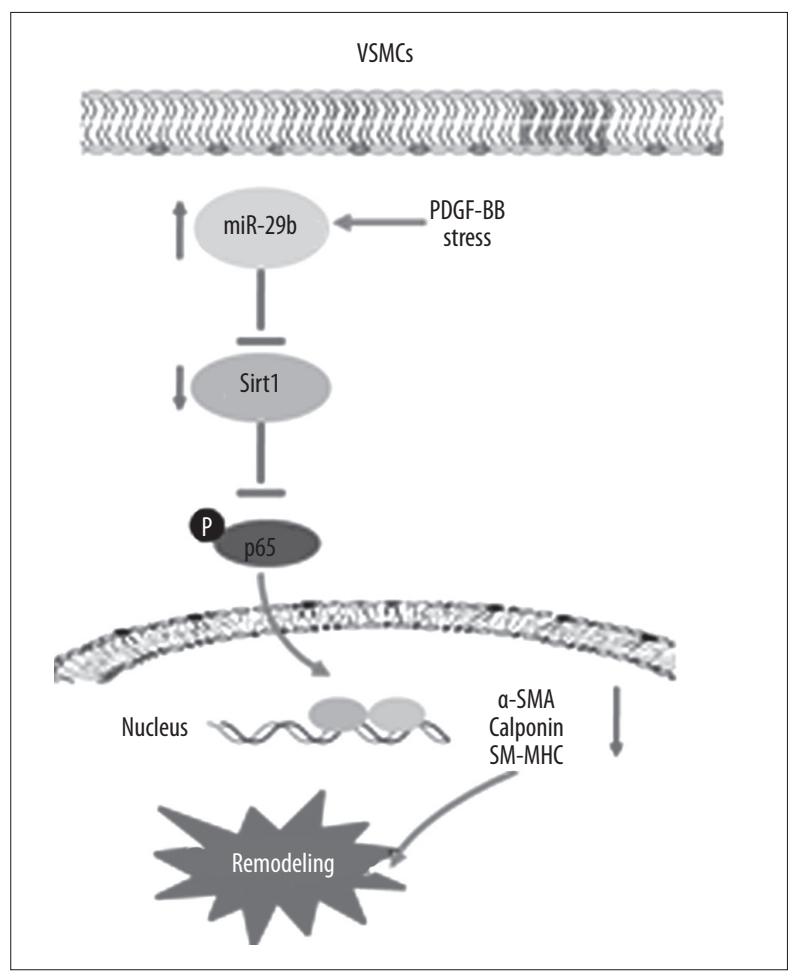

\section{References:}

1. Wang G, Jacquet L, Karamariti E, Xu Q: Origin and differentiation of vascular smooth muscle cells. J Physiol, 2015; 593: 3013-30

2. Regent A, Ly KH, Lofek $\mathrm{S}$ et al: Proteomic analysis of vascular smooth muscle cells in physiological condition and in pulmonary arterial hypertension: Toward contractile versus synthetic phenotypes. Proteomics, 2016; 16: 2637-49

3. Sandison ME, Dempster J, McCarron JG: The transition of smooth muscle cells from a contractile to a migratory, phagocytic phenotype: Direct demonstration of phenotypic modulation. J Physiol, 2016; 594: 6189-209

4. Saleh AT, Iratni R, Eid AH: Anti-atherosclerotic plants which modulate the phenotype of vascular smooth muscle cells. Phytomedicine, 2016 23: $1068-81$

5. Chappell J, Harman JL, Narasimhan VM et al. Extensive proliferation of a subset of differentiated, yet plastic, medial vascular smooth muscle cells contributes to neointimal formation in mouse injury and atherosclerosis models. Circ Res, 2016; 119: 1313-23

6. Zhang Y, Menon NV, Li C et al: The role of bifurcation angles on collective smooth muscle cell biomechanics and the implication in atherosclerosis development. Biomater Sci, 2016; 4: 430-38

7. Belo VA, Guimaraes DA, Castro MM: Matrix metalloproteinase 2 as a potential mediator of vascular smooth muscle cell migration and chronic vascular remodeling in hypertension. J Vasc Res, 2015; 52: 221-31

8. Helenius MH, Vattulainen S, Orcholski $M$ et al: Suppression of endothelial CD39/ENTPD1 is associated with pulmonary vascular remodeling in pulmonary arterial hypertension. Am J Physiol Lung Cell Mol Physiol, 2015; 308: L1046-57

9. Coll-Bonfill N, de la Cruz-Thea B, Pisano MV, Musri MM: Noncoding RNAs in smooth muscle cell homeostasis: Implications in phenotypic switch and vascular disorders. Pflugers Arch, 2016; 468: 1071-87

10. Rotllan N, Price N, Pati P et al: microRNAs in lipoprotein metabolism and cardiometabolic disorders. Atherosclerosis, 2016; 246: 352-60

11. Wang TM, Chen KC, Hsu PY et al: microRNA let-7g suppresses PDGF-induced conversion of vascular smooth muscle cell into the synthetic phenotype. J Cell Mol Med, 2017; 21: 3592-601
Figure 7. Schematic layout of showing miR-29b modulates the transformation of VSMCs phenotype through SIRT1. In the presence of PDGF-BB, miR-29b is upregulated to inhibit Sirt1, which further blocks NF-KB activation, resulting in contractile-synthetic transformation in phenotypes of VSMCs and vascular remodeling.

transfected with miR-29b inhibitor, indicating the potential role of Sirt1 in phenotypic switch of VSMCs induced by miR-29b.

\section{Conclusions}

In conclusion, miR-29b regulates the phenotypic switch of VSMC, which is mediated by PDGF-BB through SIRT1 and is a potential target in treatment of vascular diseases.

\section{Conflict of interests}

None.

12. Gareri $C$, laconetti $C$, Sorrentino $S$ et al: miR-125a-5p modulates phenotypic switch of vascular smooth muscle cells by targeting ETS-1. J Mol Biol, 2017; 429: 1817-28

13. Zong Y, Wu P, Nai C et al: Effect of microRNA-30e on the behavior of vascular smooth muscle cells via targeting ubiquitin-conjugating enzyme E2I. Circ J, 2017; 81: 567-76

14. Iaconetti C, De Rosa S, Polimeni A et al: Down-regulation of miR-23b induces phenotypic switching of vascular smooth muscle cells in vitro and in vivo. Cardiovasc Res, 2015; 107: 522-33

15. Tang Y, Yu S, Liu Y et al: MicroRNA-124 controls human vascular smooth muscle cell phenotypic switch via Sp1. Am J Physiol Heart Circ Physiol, 2017; 313: H641-49

16. Cao J, Zhang K, Zheng J, Dong R: MicroRNA-146a and -21 cooperate to regulate vascular smooth muscle cell proliferation via modulation of the Notch signaling pathway. Mol Med Rep, 2015; 11: 2889-95

17. Sun H, Cai S, Zhang M et al: MicroRNA-206 regulates vascular smooth mus cle cell phenotypic switch and vascular neointimal formation. Cell Biol Int 2017; 41: 739-48

18. Dong N, Wang W, Tian J et al: MicroRNA-182 prevents vascular smooth muscle cell dedifferentiation via FGF9/PDGFR beta signaling. Int J Mol Med, 2017; 39: 791-98

19. Li Z, Margariti A, Wu Y et al: MicroRNA-199a induces differentiation of induced pluripotent stem cells into endothelial cells by targeting sirtuin 1 . Mol Med Rep, 2015; 12: 3711-17

20. Mizuno K, Seki N, Mataki H et al: Tumor-suppressive microRNA-29 family inhibits cancer cell migration and invasion directly targeting LOXL2 in lung squamous cell carcinoma. Int J Oncol, 2016; 48: 450-60

21. Xu J, Li L, Yun HF, Han YS: MiR-138 promotes smooth muscle cells proliferation and migration in $\mathrm{db} / \mathrm{db}$ mice through down-regulation of SIRT1. Biochem Biophys Res Commun, 2015; 463: 1159-64

22. Yoshida T, Yamashita M, Horimai $C$, Hayashi $M$ : Smooth muscle-selective inhibition of nuclear factor-kappaB attenuates smooth muscle phenotypic switching and neointima formation following vascular injury. J Am Heart Assoc, 2013; 2: e230 
23. Zhang MJ, Zhou Y, Chen L et al: An overview of potential molecular mechanisms involved in VSMC phenotypic modulation. Histochem Cell Biol, 2016; 145: $119-30$

24. Uryga AK, Bennett MR: Ageing induced vascular smooth muscle cell senescence in atherosclerosis. J Physiol, 2016; 594: 2115-24

25. Veyssier-Belot C, Cacoub P: Role of endothelial and smooth muscle cells in the physiopathology and treatment management of pulmonary hypertension. Cardiovasc Res, 1999; 44: 274-82

26. Hadrava V, Kruppa U, Russo RC et al: Vascular smooth muscle cell proliferation and its therapeutic modulation in hypertension. Am Heart J, 1991; 122: 1198-203

27. Li H, Xiang Y, Fan LJ et al: Myocardin inhibited the gap protein connexin 43 via promoted miR-206 to regulate vascular smooth muscle cell phenotypic switch. Gene, 2017; 616: 22-30

28. Wei X, Hou X, Li J, Liu Y: miRNA-181a/b regulates phenotypes of vessel smooth muscle cells through serum response factor. DNA Cell Biol, 2017, 36: 127-35

29. Sun L, Bai Y, Zhao R et al: Oncological miR-182-3p, a novel smooth muscle cell phenotype modulator, evidences from model rats and patients. Arterioscler Thromb Vasc Biol, 2016; 36: 1386-97

30. Zhang YN, Xie BD, Sun L et al: Phenotypic switching of vascular smooth muscle cells in the 'normal region' of aorta from atherosclerosis patients is regulated by miR-145. J Cell Mol Med, 2016; 20: 1049-61
31. Zhao W, Zhao SP, Zhao YH: MicroRNA-143/-145 in cardiovascular diseases. Biomed Res Int, 2015; 2015: 531740

32. Chen S, Dong S, Li Z et al: Atorvastatin calcium inhibits PDGF-betabetainduced proliferation and migration of VSMCs Through the GO/G1 cell cycle arrest and suppression of activated PDGFRbeta-PI3K-Akt signaling cascade. Cell Physiol Biochem, 2017; 44: 215-28

33. Li W, Zhi W, Liu F et al: Paeoniflorin inhibits VSMCs proliferation and migration by arresting cell cycle and activating HO-1 through MAPKs and NFkappaB pathway. Int Immunopharmacol, 2017; 54: 103-11

34. Li K, Pan J, Wang J et al: MiR-665 regulates VSMCs proliferation via targeting FGF9 and MEF2D and modulating activities of Wnt/beta-catenin signaling. Am J Transl Res, 2017; 9: 4402-14

35. Liu $P$, Su J, Song $X$, Wang S: miR-92a regulates the expression levels of matrix metalloproteinase 9 and tissue inhibitor of metalloproteinase 3 via sirtuin 1 signaling in hydrogen peroxide-induced vascular smooth muscle cells. Mol Med Rep, 2017; 17: 1041-48

36. Kitada M, Ogura Y, Koya D: The protective role of Sirt1 in vascular tissue: Its relationship to vascular aging and atherosclerosis. Aging (Albany NY) 2016; 8: 2290-307

37. Hwang JS, Ham SA, Yoo T et al. Sirtuin 1 mediates the actions of peroxisome proliferator-activated receptor delta on the oxidized low-density lipoprotein-triggered migration and proliferation of vascular smooth muscle cells. Mol Pharmacol, 2016; 90: 522-29 